Check for updates

Cite this: RSC Adv., 2019, 9, 13494

\title{
Active targeting drug-gold nanorod hybrid nanoparticles for amplifying photoacoustic signal and enhancing anticancer efficacy $\dagger$
}

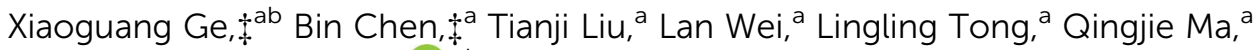 \\ Shi Gao*a and Jibin Song (D)*b
}

\begin{abstract}
The development of theranostic nanomaterials with limited side effects and increased therapeutic efficacy is a promising approach for cancer imaging and therapy. In the present study, the development of a multifunctional metal-organic hybrid nanoparticle (NP) with enhanced photoacoustic (PA) imaging performance able to be actively uptaken by cancer cells for synergistic chemo-photothermal cancer therapy was reported. The theranostic NP was composed through the coordination effect between an ultrasmall gold nanorod (AuNR), a thick coating layer of the organic near-infrared dye IR780, and the anticancer drug doxorubicin (DOX), named AuNR@IR780/DOX-RGD-PEG. In addition, the theranostic NP surface was conjugated with targeting ligand RGD and a protective PEG shell, where the PEG played a role in concealing or exposing the RGD for specific targeting of the NPs to the cancer cells. The theranostic NP demonstrated a greatly enhanced PA imaging signal compared to AuNR or IR780, due to the fact that the electromagnetic field of the AuNR increased the light absorption efficiency of the IR780 coating based on the theoretical simulation results. Furthermore, the "Trojan-horse" active targeting strategy not only increased the uptake of NPs by tumor cells, but also decreased the non-specific uptake by healthy cells, thus limiting the side effects. This study developed a smart theranostic NP for enhanced cancer PA imaging and specific cancer therapy.
\end{abstract}

\author{
Received 1st March 2019 \\ Accepted 17th April 2019 \\ DOI: 10.1039/c9ra01547g \\ rsc.li/rsc-advances
}

\section{Introduction}

Photoacoustic (PA) imaging is a modern and promising biomedical imaging technique, which exhibits several merits including deep penetration effect, super-high spatial resolution, non-invasive imaging, and nonionizing radiation. ${ }^{1-3}$ The main mechanism of PA imaging is the thermal expansion effect, which is safe and widely applied in the biomedical imaging field. ${ }^{4}$ During PA laser irradiation, the PA imaging contrast agent absorbs the light energy and converts it into thermal energy, generating a PA pressure wave within the agent. ${ }^{3,5,6}$ The PA pressure usually determines the resulting acoustic intensity, as well as the PA bioimaging performance. Thus, an excellent PA imaging agent should be a light absorber with high light-to-heat conversion efficiency. ${ }^{7,8}$

In recent years, in order to improve the PA imaging quality, a variety of nanomaterials have been explored as PA imaging

\footnotetext{
${ }^{a}$ Departments of Nuclear Medicine, China-Japan Union Hospital of Jilin University, Changchun, Jilin, 130033, China. E-mail: gaoshi@jlu.edu.cn

${ }^{b} \mathrm{MOE}$ Key Laboratory for Analytical Science of Food Safety and Biology, College of Chemistry, Fuzhou University, Fuzhou 350108, China. E-mail: jibinsong@fzu.edu.cn $\dagger$ Electronic supplementary information (ESI) available. See DOI: 10.1039/c9ra01547g

$\ddagger$ These authors contributed equally to this work.
}

agents. ${ }^{9-11}$ Among them, gold nanocrystals and organic dyes with light-absorbing properties have been widely investigated and applied in the field of biomedical imaging., ${ }^{912-16}$ When laser irradiation, a strong electromagnetic field on the gold nanocrystal surface is generated, which has the potential to improve the light absorption efficiency of the light absorbers. ${ }^{17-21}$ However, little has been reported on the interaction between organic dyes and gold nanocrystals upon laser irradiation. ${ }^{2,3,6,17,22}$ Gold nanorods (AuNR) and IR780 with the maximum absorption wavelength in the near infrared region (NIR) are usually used as PA imaging agents. In the present study, the optical interaction effect and the enhanced PA imaging performance of an AuNR surface coated with a thick layer of IR780 molecules was systematically investigated.

Apart from developing a cancer imaging modality to improve therapeutic efficacy, a variety of targeting ligands was developed in order to increase the tumor uptake efficiency of drug-loaded nanoparticle (NP). In recent years, for example, peptides and proteins (mainly antibodies or their fragments), small molecules (folic acid), and nucleic acids (aptamers) have been modified onto drug nanocarrier surfaces for active targeting. ${ }^{18,23}$ Arginine-glycine-aspartic acid (RGD), a widely used targeting ligand, has been modified onto many inorganic nanoparticles (e.g., gold NP, AuNR, and iron oxide NP), organic nanoparticles, or polymeric micelles, showing active targeting ability to 
positive $\alpha_{v} \beta_{3}$-expressing cancer cells. ${ }^{24-28}$ Another big challenge of active targeting has been the non-specific binding between the targeting molecules and non-target molecules expressed on healthy cells, usually leading to non-specific uptake of targeting ligand-modified nanoparticles by healthy or non-cancerous cells. $^{19,21,25}$ Therefore, it is highly significant to develop a nanoplatform able to protect the targeting ligand before entering into the tumor region and expose it only after entering the tumor. ${ }^{29,30}$

Herein, an AuNR coated with a PA activating organic nearinfrared dye IR780 and the anticancer drug doxorubicin (DOX) (AuNR@IR780/DOX) with high PA imaging performance for cancer imaging and therapy was developed, as displayed in Fig. 1. Due to the fact that targeting molecules may bind to nontarget healthy cells, a theranostic nanoparticle modified with targeting ligands may be uptaken by healthy cells, leading to side effects and limited therapy efficacy against the tumor. In order to minimize the uptake of healthy cells, a theranostic NP with accurate targeting ability to tumor cells was designed, based on AuNR@IR780/DOX NPs for PA imaging-guided synergistic chemo-photothermal cancer therapy. Briefly, a targeting ligand of RGD peptides modified with polyphenol was conjugated on the NP surface, and methoxy-poly(ethyleneglycol) Meo-PEG-polyphenlos ( $\mathrm{MW}=5 \mathrm{kDa}$ ) with a $\mathrm{pH}$-responsive $\beta$ thiopropionate bond was also conjugated on the NP surface through the coordination effect, forming the theranostic AuNR@IR780/DOX-RGD-PEG NPs. During the circulation of the theranostic NPs in the bloodstream (pH 7.4), the PEG shell prevents the hidden RGD from contacting with healthy cells, reducing non-specific uptake. After the theranostic NPs enter into the tumor tissue, the PEG shell is shielded by the tumor

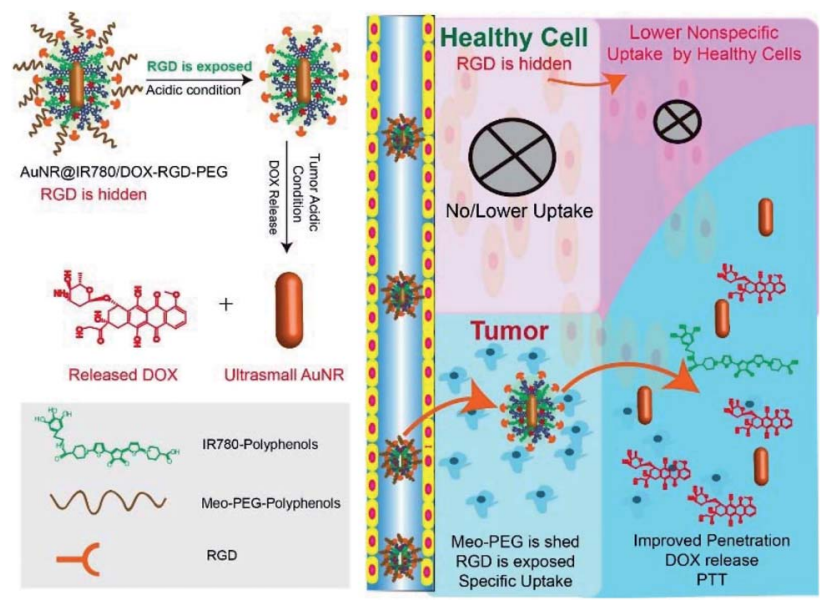

Fig. 1 Scheme illustration of the multifunctional AuNR@IR780/DOXRGD-PEG composite nanoparticles and the mechanism of preventing nonspecific uptake by heathy cells with non-specific receptors. The Meo-PEG shell protects the hidden RGD when the hybrid NP is circulating in the bloodstream, preventing RGD from binding to the normal healthy cells. However, Meo-PEG is shed in the tumor because the $\mathrm{pH}$-labile $\beta$-thiopropionate linker is broken that was caused by the acidic tumor microenvironment. Then, the RGD ligand is exposed and is able to specifically bind to the U87 MG tumor cells with $\alpha_{v} \beta_{3}$ expressed on the cell surface. acidity, enabling the exposed RGD to effectively bind to the $\alpha_{\mathrm{v}} \beta_{3}$ positive cancer cells. Thus, the theranostic NPs can be precisely uptaken by the tumor cells.

\section{Experimental section}

\section{Materials}

All compounds used were purchased from Sigma Aldrich. These compounds are doxorubicin hydrochloride (DOX), sodium borohydride $\left(\mathrm{NaBH}_{4}\right)$, ascorbic acid (AA), Meo-PEG-OH (MW = 5000), mercapto acetic acid, dopamine hydrochloride, hexadecyl trimethyl ammonium bromide (CTAB), silver nitrate, acryloyl chloride (AC), triethylamine (TEA), hoechst 3342, hydrochloric acid, tetrachloroaurate(III) trihydrate $\left(\mathrm{KAuCl}_{4}{ }^{-}\right.$ $\cdot 3 \mathrm{H}_{2} \mathrm{O}$ ). All water used, unless specified otherwise, is deionized water.

\section{Preparation of the AuNR@IR780/DOX-RGD-PEG NPs}

The as-made ultrasmall AuNR coated with CTAB (ESI $\dagger$ ) we firstly modified with SH-PEG $(\mathrm{Mn}=20 \mathrm{kD})$ to remove CTAB. The asprepared PEG modified AuNR (AuNR@PEG), DOX and polyphenol modified IR780 with pre-determined molar ratio were dissolved in DMSO $(500 \mu \mathrm{L})$, and then excess $\mathrm{FeCl}_{3}\left(2 \mathrm{mg} \mathrm{mL} \mathrm{m}^{-1}\right.$ in water) was slowly added to the mixed solution. After stirring for $15 \mathrm{~min}$, D.I water was added into the solution drop-by-drop to trigger the formation of the hybrid AuNR@IR780/DOX NPs. In the following, the mixture of RGD-polyphenols and Meo-PEGpolyphenols was added into the solution. After reaction for $5 \mathrm{~h}$ the PEG and RGD (ESI $\dagger$ ) conjugated AuNR@IR780/DOX-RGDPEG NP was prepared, which was further purified by centrifugation $(8000 \mathrm{~g}$ ) for $5 \mathrm{~min}$. The as-prepared theranostic NP was stored in water at $4{ }^{\circ} \mathrm{C}$ for further use.

\section{Cellular Uptake of the Hybrid AuNR@IR780/DOX-RGD-PEG NPs}

The U87MG cancer cells maintained in DMEM medium with $10 \%$ FBS was employed as the target cell line for the following in vitro cell experiments. U87MG cancer cells were seeded on 12well glass plate with a density of $1 \times 10^{4}$ cells per well, and further incubated in growth medium at $37{ }^{\circ} \mathrm{C}$ with $5 \% \mathrm{CO}_{2}$. After $24 \mathrm{~h}$ incubation, the theranostic NPs pre-incubated at $\mathrm{pH}$ 6.5 or $\mathrm{pH} 7.4$ solution were added into the well. After incubation for $1 \mathrm{~h}$, the samples were removed, and the cells were washed by fresh PBS. Afterwards, fresh DMEM medium was added into the well. Fluorescence imaging of the DOX in the cells was acquired by using laser scanning confocal microscopy (CLSM).

\section{In vitro cancer imaging of the AuNR@IR780/DOX-RGD-PEG NPs}

U87MG cancer cells were seeded in a 8-well Lab-Tek coverglass slides (25, 000 cells per well). For cancer cell imaging, the samples were added in the plate. After incubation for $1 \mathrm{~h}$, the samples and medium were removed, the cells were washed with fresh PBS. The cells were incubated in fresh medium for different times. At the same time, the fluorescence imaging of 
the cells was obtained by CLSM at $1 \mathrm{~h}$ timepoint, and $3 \mathrm{~h}$ and $5 \mathrm{~h}$ post-incubation.

\section{In vitro cancer therapy of the AuNR@IR780/DOX-RGD-PEG NPs}

For cancer cell therapy experiment, the U87MG cells were cultured in 96-well plate. After incubation for $24 \mathrm{~h}$, the samples were added in the well for $1 \mathrm{~h}$. Afterwards, the medium was removed and replaced with fresh medium. The cells were exposed with $808 \mathrm{~nm}$ laser $\left(0.25 \mathrm{~W} \mathrm{~cm}^{-2}\right)$ for $5 \mathrm{~min}$. After the cells were incubated for $24 \mathrm{~h}$, the cell viability was tested using the CCK-8 assay.

\section{In vivo photoacoustic imaging}

All animal experiments were performed in compliance with the Guide for the Care and Use of Laboratory Animals (Ministry of Science and Technology of China, 2006) and approved by the Institutional Animal Care and Use Committee of Fujian Medical University. The U87MG tumor was grown by inoculating the U87MG cells $\left(100 \mu \mathrm{L} 1 \times 10^{6}\right)$ into the right shoulder of each nude mice ( 8 weeks old, female). When the tumor volume is $\sim 60 \mathrm{~mm}^{3}$, the theranostic NP in PBS $(100 \mu \mathrm{L})$ was injected intravenously into the U87MG tumor-bearing mice through tail vein. Afterwards, dual model of PA and US images and the PA signal intensity of the tumor were recorded by using the PA equipment (2100 LAZR system, Visual Sonic Vevo) at different post-injection times.

\section{In vivo synergistic chemo-photothermal therapy}

Thirty tumor-bearing nude mice were divided into 6 groups. When the tumor volume reach $\sim 60 \mathrm{~mm}^{3}$, the mice was treated by (1) PBS, (2) free DOX, (3) AuNR@IR780/DOX-RGD, (4) AuNR@IR780/DOX-PEG NPs, (5) AuNR@IR780/DOX-RGD-PEG NPs, (6) AuNR@IR780/DOX-RGD-PEG NPs with laser irradiation, respectively. Before and after cancer therapy, the tumor length and width of each tumor-bearing mice was measured every two days, and the tumor volume was calculated and recorded. At mean time, the body weight of the mice in each group was recorded at an interval day. At day 18 post-treatment, the treated mice were sacrificed, and the main organs (heart, liver, lung, spleen, kidney), and tumors of one mice in each groups were collected and stored in $4 \%$ paraformaldehyde solution for H\&E staining. The images of them were acquired by using microscopy.

\section{Results and discussion}

Preparation of hybrid core-shell AuNR@IR780/DOX-RGD-PEG NPs

AuNRs with the length of $\sim 15 \mathrm{~nm}$ and width of $\sim 3 \mathrm{~nm}$ were firstly synthesized using a seed-less method previously reported by our group (Fig. 2a, Experimental procedures). ${ }^{4,31}$ The theranostic AuNR@IR780/DOX-RGD-PEG NPs were prepared by selfassembly of AuNR, polyphenol modified IR780, and DOX in DMSO, in the presence of water and ferric ions $\left(\mathrm{Fe}^{3+}\right)$ (Experimental procedures). ${ }^{32}$ Afterwards, the polyphenol modified
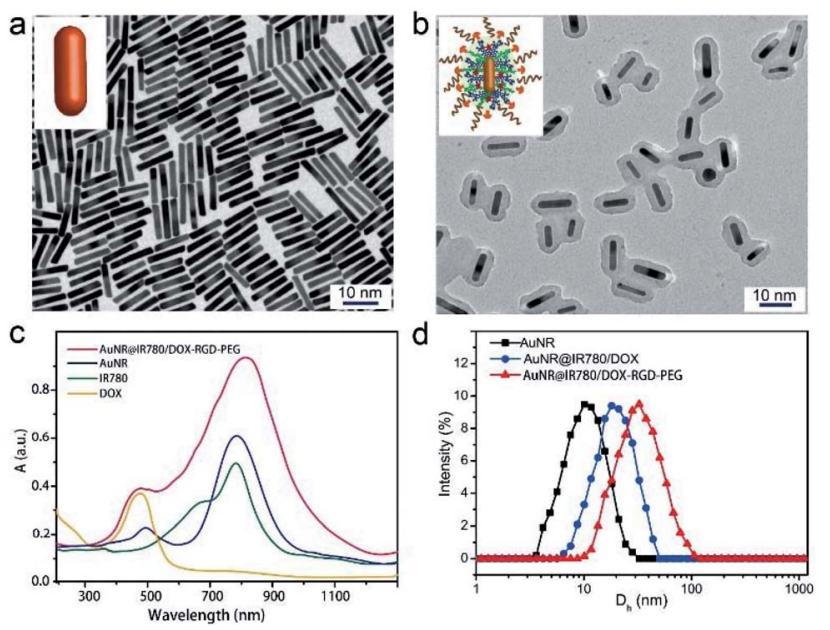

Fig. 2 TEM images of ultrasmall AuNR (a) and theranostic AuNRaIR780/DOX-RGD-PEG NPs (b). (c) UV-vis spectra of the AuNR, IR780, DOX and AuNR@IR780/DOX-RGD-PEG NPs in aqueous solution. (d) Hydrodynamic diameter of AuNR, AuNR@IR780/DOX and AuNR@IR780/DOX-RGD-PEG NPs in aqueous solution.

RGD and Meo-PEG-polyphenol with an acid-labile $\beta$-thiopropionate linker were added in the mixed solution, forming the theranostic NPs. As it can be seen in the TEM images (Fig. 2b), a thick layer of IR780 and DOX was clearly observable being closely surrounded on the AuNR surface. The coated amount of the IR780 and DOX can be facilely adjusted by changing the reaction time or the initial added amount of them. A thicker layer of the IR780 and DOX was coated on the AuNR surface by prolonging the reaction time (Fig. S1a †). We further employed XPS to characterize the composition of the hybrid NP. No Au element signal was observed in the XPS spectra of the hybrid NP, suggesting a thick organic layer was coated on the AuNR surface (Fig. S2 †).

The characteristic absorption peaks of IR780 at $780 \mathrm{~nm}$ and DOX at $490 \mathrm{~nm}$ were obviously apparent in the UV-vis spectrum of the theranostic NPs as compared to that of AuNR (Fig. 2c). Moreover, the maximum localized surface plasmon resonance (LSPR) peak of the AuNR was consistent with that of IR780. Based on the dynamic light scattering (DLS) results, the coated layer thickness was about $5 \mathrm{~nm}$, while the diameter of the NP was $\sim 27 \mathrm{~nm}$, suggesting a high loading content of IR780 and DOX. Furthermore, after conjugation with RGD and PEG, the diameter of the as-made NPs was further increased to $\sim 35 \mathrm{~nm}$, suggesting a successful conjugation (Fig. 2d). The zeta potential of the theranostic NP is $\sim 24.5 \mathrm{mV}$ due to negative charged PEG was coated on the NP's surface (Fig. S3†).

\section{Enhanced photothermal and photoacoustic performances of smart hybrid NPs}

In the next step, the optical interaction between AuNR and the coated NIR dye IR780 under laser irradiation was investigated. The temperature variations of the AuNR@IR780/DOX, IR780, and AuNR solution with the same optical density at $808 \mathrm{~nm}$ $\left(\mathrm{OD}_{808}\right)$ after laser irradiation at $0.25 \mathrm{~W} \mathrm{~cm}^{-2}$ for $5 \mathrm{~min}$ were 
tested and are displayed in Fig. 3a. The temperature increment of the theranostic NPs was $60^{\circ} \mathrm{C}$, which was higher than that of both IR780 $\left(\sim 19.5{ }^{\circ} \mathrm{C}\right)$ and AuNR $\left(\sim 14{ }^{\circ} \mathrm{C}\right)$. Furthermore, the theranostic NPs exhibited concentration-dependent photothermal properties, which demonstrated a linear increment with the increasing NP concentration (Fig. 3b). Even at very low concentrations, the temperature increased more than $20{ }^{\circ} \mathrm{C}$. The greatly enhanced photothermal properties of the theranostic NP were attributed to the electromagnetic field of the AuNR, which increased the light absorption efficiency of the coated IR780.

Both in vitro and in vivo PA imaging quality is directly related to the photothermal properties of the contrast agent. ${ }^{33-35}$ An excellent PA imaging contrast agent not only should be biocompatible but should also have high photothermal conversion efficiency. ${ }^{\mathbf{1 4 , 3 5}}$ In order to evaluate the PA imaging performance of the hybrid NP as well as the AuNR and IR780, the PA signal intensities were measured at different optical density at $808 \mathrm{~nm}\left(\mathrm{OD}_{808}\right)$ values under laser illumination. As it can be observed in Fig. 3c, the hybrid NP showed brighter PA signals than those of the AuNR and IR780 at the same OD $_{808}$ value. At a low $\mathrm{OD}_{808}$ value, the hybrid NPs produced an ultrabright PA signal, which can be vital and useful for the following in vivo biomedical imaging applications. The PA intensity of the hybrid NP was about 3 times higher than that of the AuNR and IR780 at the same $\mathrm{OD}_{808}$ value, and was found to linearly increase with the increasing $\mathrm{OD}_{808}$ value (Fig. $3 \mathrm{~d}$ ). The theranostic NP showed high stability after PA imaging laser irradiation for 10 times (Fig. S4†).

The photothermal and PA performances of samples were associated with their photothermal conversion efficiency, which is a standard for physical-optical property evaluation..$^{10,33,36,37}$ In order to calculate the photothermal conversion efficiency of the hybrid NP, the heating and cooling curves of the hybrid NPs were also recorded (Fig. 3e and f). Based on the experimental results, the photothermal conversion efficiency was found to be $67.5 \%$, which was much higher than that of AuNR $(22 \%) .{ }^{38}$ The
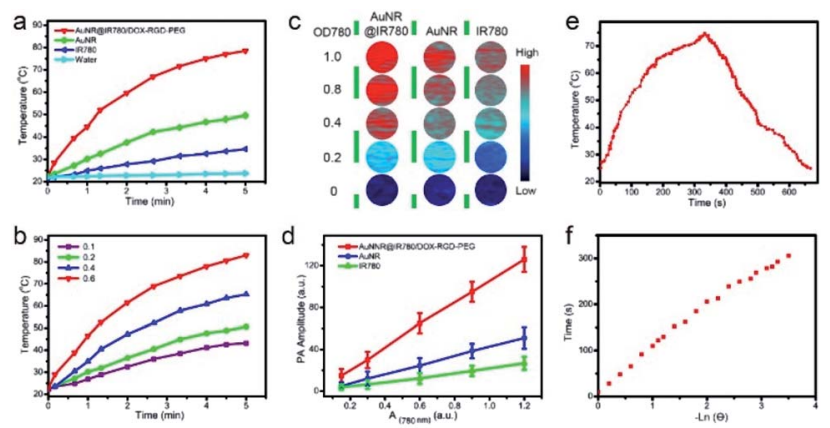

Fig. 3 (a) Temperature increase of different samples in water irradiated with $808 \mathrm{~nm}$ laser. (b) Temperature variation of AuNR@IR780/ DOX-RGD-PEG NPs with different optical density at $808 \mathrm{~nm}$ upon $808 \mathrm{~nm}$ laser exposure. PA images (c) and PA amplitude (d) of the samples with different optical density values at $808 \mathrm{~nm}$. (e) Temperature variation of the AuNR@IR780/DOX-RGD-PEG NPs in water during and after laser irradiation. (f) The cooling curve of the theranostic NP after 5 min laser irradiation. electromagnetic field distribution around the AuNR surface accounted for the greatly enhanced excitation of the light-toheat conversion efficiency. The reported core-shell AuNRIR780 hybrid NP, not only combined two kinds of PA imaging agent (AuNR and IR780) in one platform, but also resulted to a more enhanced PA imaging signal than the isolated AuNR and IR780 signals.

\section{Theoretical study of the mechanism of the enhanced physical-optical properties}

In order to investigate the mechanism of enhanced photothermal conversion efficiency of the hybrid NP and further verify the experimental results, the electromagnetic field (EM) distribution and its influence to the light absorption property of the coated IR780 layer was calculated. ${ }^{39}$ As it can be seen in Fig. $4 \mathrm{a}$ and b, the EM field was distributed around the surface of the AuNR, especially at its ends. Moreover, the EM field of the AuNR@IR780/DOX NPs was found 3.5 times higher than that of the bare AuNRs (Fig. 4c and d), and the calculated data was consistent with the amplified PTT effect and PA imaging performance of the hybrid AuNR@IR780/DOX NPs. The amplified EM field distribution (red colored) was produced due to the increased light absorption efficiency and properties of the hybrid NP depending on their special nanostructures and types of nanomaterial. Single AuNRs exhibited relatively low EM field distributions owing to the lack of light absorption of the IR780 in the $750 \mathrm{~nm}$ wavelength. The calculated absorption spectra of the hybrid NP is consistent with the experimental data (Fig. 4e and $\mathrm{f}$ ). Taken together, these results confirmed that the strong EM field is important to increase the light absorption efficiency and eventually enhance the PA signal.

\section{Anticancer drug loading and stimuli-responsive drug release}

Afterwards, the DOX loading ability of the theranostic NPs was investigated by changing the initial mass ratio between the DOX and the AuNR during the preparation process of the NPs. ${ }^{40-42}$ As shown in Fig. 5a, the DOX loading content increased with the initial feeding ratio of DOX and AuNR, and reached $\sim 20 \%$ when

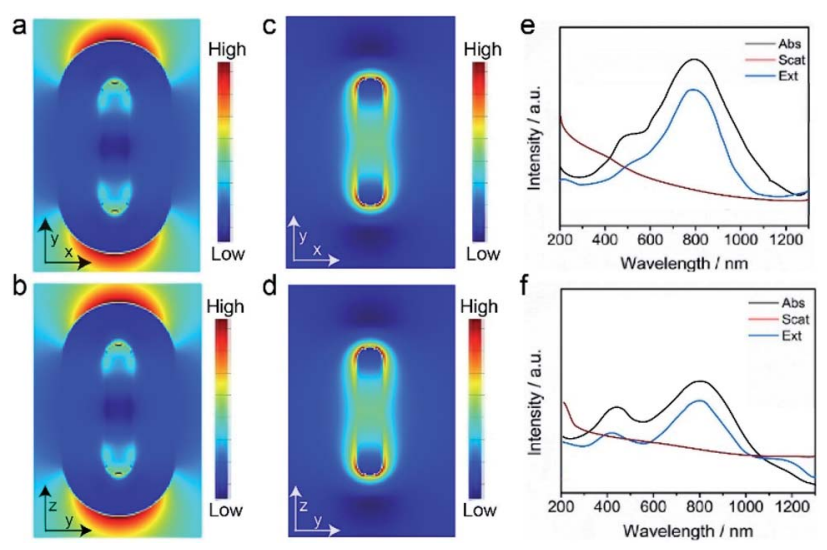

Fig. 4 Theoretical calculation of the EM filed of the AuNR@IR780/ $\operatorname{DOX}(a$ and $b$ ) and AuNR ( $c$ and d), and absorption, scattering and extinction spectra of AuNR(IR780/DOX (e) and AuNR (f). 

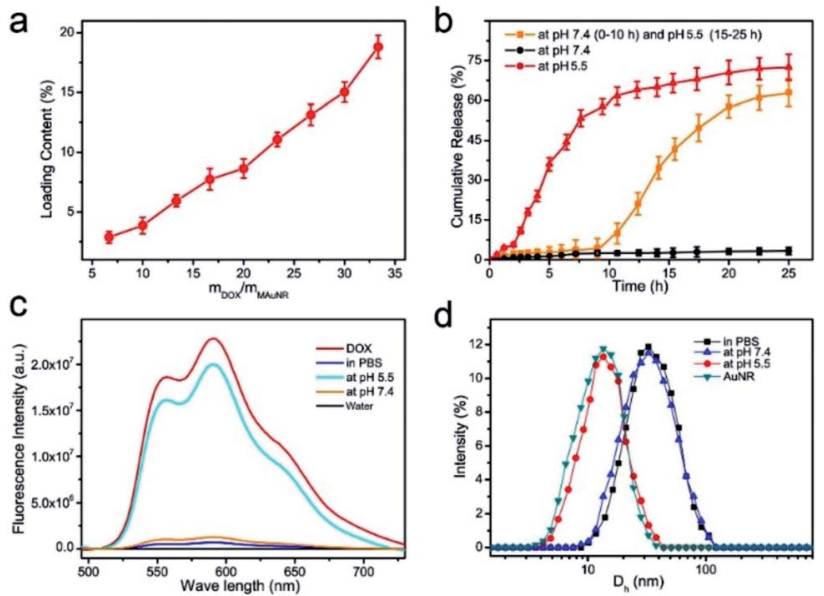

Fig. 5 (a) The DOX loading content in the theranostic NPs. (b) In vitro DOX release profiles of the theranostic NPs in different conditions. (c) Fluorescence spectra of free DOX and the theranostic NPs in PBS, or in aqueous solution at $\mathrm{pH} 5.5$ and $\mathrm{pH}$ 7.4. (d) Hydrodynamic diameter distribution of the AuNR and theranostic NPs in PBS, and in water at pH 5.5 and $\mathrm{pH} 7.4$

the ratio was 35 . The thick organic layer of IR780 and DOX was not observed in the TEM images of the AuNR@IR780/DOXRGD-PEG NPs after treatment with acidic solution ( $\mathrm{pH} 5.5)$ for $15 \mathrm{~h}$ (Fig. $\mathrm{S} 1 \mathrm{~b}_{\dagger}^{\dagger}$ ). Moreover, the $\mathrm{Au}_{4 \mathrm{f}}$ signal was also detected when the DOX was released and removed from the surface of the theranostic NP (Fig. S2 $\dagger$ ), confirming the organic layer was dis-assembled. In the following step, the DOX release behavior of the theranostic NPs triggered by the acidic environment was examined. Due to the fact that the coordination and $\pi-\pi$ stacking effects between polyphenols and $\mathrm{Fe}^{3+}$ are $\mathrm{pH}-$ responsive, the theranostic NPs would disassemble in the acidic solution, leading to DOX release. Thus, the DOX release of the theranostic NP was tested at $\mathrm{pH} 5.5$ and 7.4. According to the results, no DOX was released from the theranostic NPs incubated in water at pH 7.4 (Fig. 5b), while when the theranostic NPs were incubated in an aqueous solution at $\mathrm{pH}$ 5.5, rapid DOX release was observed, and over $70 \%$ of the DOX was released at the $15 \mathrm{~h}$ time point. These results confirmed that the theranostic NPs exhibit high pH-responsive ability, leading to PEG organic shell dissolving and DOX release. To further verify this hypothesis, the theranostic NPs were initially incubated in an aqueous solution at $\mathrm{pH} 7.4$ for $10 \mathrm{~h}$, and then the $\mathrm{pH}$ value of the aqueous solution was tuned to 5.5 by adding $\mathrm{HCl}(1 \mathrm{M})$ solution. An interesting finding was that the theranostic NP was stable and no DOX was released between 0-10 h, whereas a burst release of DOX was recorded after the $\mathrm{pH}$ value was changed to 5.5. Moreover, the theranostic NP is also stable with negligible DOX release in the presence of $\mathrm{GSH}$ or $\mathrm{H}_{2} \mathrm{O}_{2}$ (Fig. S5†).

Due to the fluorescence quenching effect of the plasmonic nanoparticle to fluorescence dye, a weak fluorescence red signal of DOX was observed in the theranostic NP aqueous solution. ${ }^{38}$ However, when the theranostic NP was treated with a $\mathrm{pH} 5.5$ aqueous solution for $15 \mathrm{~h}$, the fluorescence signal of DOX was enhanced, indicating that DOX was released from the AuNR surface, as it can be observed in Fig. 5c. Moreover, after incubation in the $\mathrm{pH} 7.4$ aqueous solution of PBS for $15 \mathrm{~h}$, a very weak fluorescence signal of DOX was detected, suggesting that DOX was still located on the theranostic NP surface. Additionally, the diameter of the theranostic NP was decreased to $\sim 15 \mathrm{~nm}$, which was consistent with the AuNR size, confirming also the dissolving of the organic shell of the theranostic NP (Fig. 5d).

\section{In vitro specific targeting and therapy with the smart theranostic NPs}

In order to investigate the precise targeting ability of the theranostic NPs to U87MG cancer cells, PEG was conjugated onto the theranostic NP surface via a $\mathrm{pH}$-responsive bond able to conceal RGD and expose it after acid-sensitive PEG shedding in the acidic tumor microenvironment. U87MG cancer cells were employed to investigate the targeting ability of the theranostic NP, since RGD can efficiently bind to $\alpha_{v} \beta_{3}$, which is exposed on the U87MG cancer cell surface. After the cells were incubated with the theranostic NP at pH 6.5 for $1 \mathrm{~h}$, a weak fluorescence signal of DOX was observed at $3 \mathrm{~h}$ and $5 \mathrm{~h}$ postincubation, because AuNR quenched the fluorescence signal of DOX (Fig. 6a). After $5 \mathrm{~h}$ incubation, a strong DOX fluorescence signal was recorded in the cell nuclei, suggesting that DOX was released from the NPs and entered the cell nuclei (Fig. 6a). On the other hand, the cells treated with theranostic

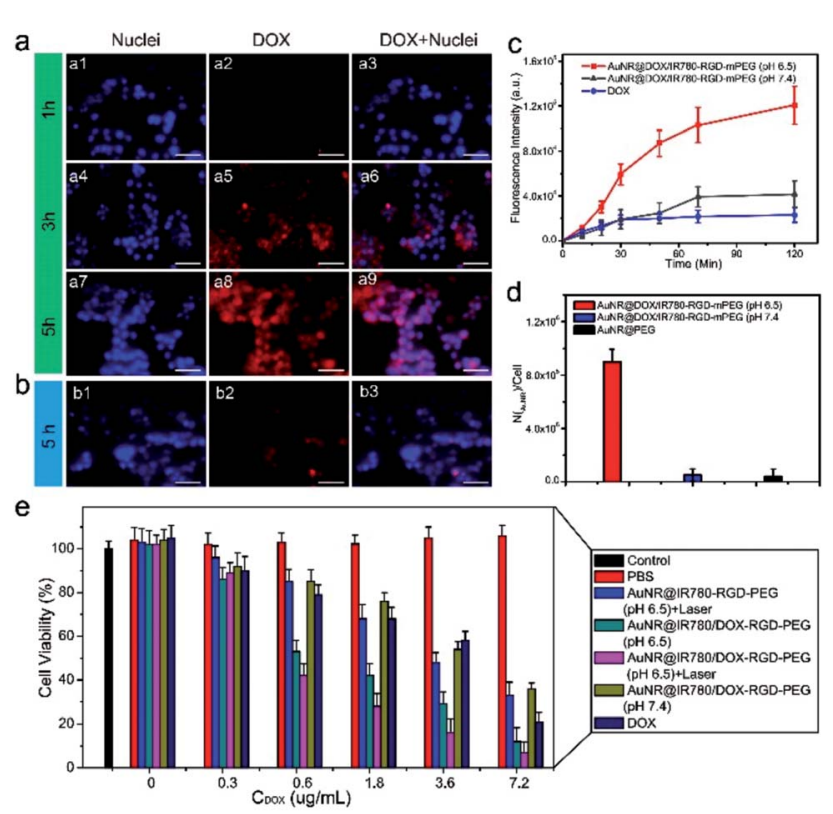

Fig. 6 In vitro fluorescence images of cancer cells after incubated with the theranostic NP at $\mathrm{pH} 6.5$ (a) and $\mathrm{pH} 7.4$ (b) cell culture solutions after 1, 3 and $5 \mathrm{~h}$ post-incubation. (blue color: cell nuclei, red color: DOX). Scale bars: $50 \mu \mathrm{m}$. (c) The average fluorescence intensity of the DOX in the cells in different treatment groups. (d) The calculated number of AuNRs uptake by each cell in different treatment groups. (e) In vitro cytotoxicity of the cancer cells incubated with different samples and with/without laser irradiation $\left(808 \mathrm{~nm}, 0.25 \mathrm{~W} \mathrm{~cm}^{-2}\right)$ for 5 min. 
NPs at $\mathrm{pH}$ 7.4, at $5 \mathrm{~h}$ min post-incubation exhibited a weaker signal than that of the cells incubated at pH 6.5 (Fig. 6b). In Fig. 6c, it can be observed that the fluorescence intensity of the cells at $\mathrm{pH} 6.5$ was about 3 times higher than that at $\mathrm{pH} 7.4$, suggesting that the PEG was removed at $\mathrm{pH} 6.5$ and the NPs with exposed RGD were uptaken more efficiently by the cancer cells. The DOX signal in the cell treated by the theranostic NPs for $1 \mathrm{~h}$ and $3 \mathrm{~h}$ and $5 \mathrm{~h}$ post-incubation also gradually increased (Fig. S6†). Additionally, inductively coupled plasma mass spectroscopy (ICP-MS) was used as an analytical approach in order to perform accurate quantitative comparisons. As presented in Fig. 6d, the number of AuNRs in the cells incubated with the theranostic NPs at pH 6.5 was $9.5 \times 10^{5}$, which was about 100 times higher than that of the cells treated with theranostic NPs at pH $7.4\left(0.5 \times 10^{5}\right)$. As control experiments, $\alpha_{\mathrm{v}} \beta_{3}$ negative MCF-7 cancer cells or U87MG cells blocked by free RGD after treated by theranostic NP at pH 6.5 showed very weak fluorescence signal of DOX, indicating low cell uptake efficiency of the theranostic NP (Fig. S7 $\dagger$ ). Moreover, the number of AuNR in the healthy cells treated with AuNR@IR780/DOX-RGD-PEG NPs is significantly less than that of the healthy cells incubated with AuNR@IR780/DOX-RGD NPs (Fig. S8†), indicating the PEG protecting shell reduced non-specific uptake of the theranostic NPs by healthy cells.

A technique for active targeting of theranostic NPs to $\alpha_{\mathrm{v}} \beta_{3^{-}}$ positive U87MG cancer cells, combined with $\mathrm{pH}$-triggered DOX release and photothermal properties, may be an excellent candidate for a cancer therapy nanoplatform. ${ }^{43}$ In the next step, the in vitro cancer therapy effect of theranostic NPs with and without laser irradiation was evaluated. Free DOX and solely laser irradiation served as the control groups. Prior to incubation with cancer cells, the AuNR@IR780/DOX-RGD-PEG NPs were incubated at $\mathrm{pH} 6.5$ or 7.4 to remove the PEG. The U87MG cell viabilities of AuNR@IR780/DOX-RGD-PEG NPs were DOX dosage-dependent, and those incubated at $\mathrm{pH} 6.5$ prior to incubation with the cells were much more toxic that those incubated at $\mathrm{pH} 7.4$ (Fig. 6d). The reason was that the PEG shedding at $\mathrm{pH}$ 6.5, led to RGD modified NPs able to be taken up by the cells, which was also consistent with the fluorescence imaging and ICP-MS results (Fig. 6c and d). In combination with laser irradiation, the viability of cells treated with AuNR@IR780/DOX-RGD-PEG NPs was further reduced, suggesting synergistic chemo-photothermal therapy. The IC50 was about $0.52 \mu \mathrm{g} \mathrm{mL} \mathrm{m}^{-1}$, which was significantly lower than the DOX-free value $\left(4.2 \mu \mathrm{g} \mathrm{mL}{ }^{-1}\right)$. The active targeting ability of the theranostic NPs after PEG shedding, combined with its excellent photothermal therapy effect, made this novel nanoplatform an excellent candidate as a cancer therapy agent.

\section{In vivo enhanced PA imaging and tumor accumulation of theranostic NPs}

In order to investigate the in vivo PA imaging effect of the AuNR@IR780/DOX-RGD-PEG NPs, the U87MG xenograft tumor model was established in nude mice. Moreover, the theranostic NPs exhibited excellent stability with size variation in physiological condition for 7 days, making it suitable for in vivo applications (Fig. S9†). When the tumor size reached $\sim 60 \mathrm{~mm}^{3}$, the theranostic NPs were intravenously injected into the tumorbearing mice through the tail vein. PA imaging and intensity of the tumor were recorded in real-time. Fig. 7a shows the ultrasound (US) and PA imaging of the tumor, where the PA signal was observed to increase with increasing post-injection times, which reached a maximum at $\sim 20 \mathrm{~h}$ post-injection. The tumor morphology, position, and size based on the PA imaging could be clearly observed. However, due to the metabolism of the NPs, the PA signal was slightly decreased after $20 \mathrm{~h}$ post-injection. Moreover, the tumor treated with AuNR@IR780/DOX-RGD showed a weaker PA signal than that of the AuNR@IR780/ DOX-RGD-PEG, because without PEG protection the in vivo circulation time was relatively shorter. The blood halfcirculation time $\left(t_{1 / 2}\right)$ in vivo of the hybrid NP is $\sim 19 \mathrm{~h}$, which is longer than that of AuNR@IR780/DOX NPs $\left(t_{1 / 2}=6.5 \mathrm{~h}\right)$ and AuNR@IR780/DOX-RGD NPs $\left(t_{1 / 2}=7.2\right.$ h) (Fig. S10 $\dagger$ ). Even though the NPs without RGD were protected by PEG, they produced a reduced PA signal in the tumor region compared to that of the AuNR@IR780/DOX-RGD-PEG NPs with PEG shedding ability, further confirming the enhanced tumor accumulation of the theranostic NPs in the tumor. The theranostic NPs could slowly penetrate into the deep tumor region based on the high resolution PA images of the tumor treated with the theranostic NPs (Fig. S11 $\dagger$ ). ${ }^{44,45}$ The recorded PA signal intensities of the three samples further confirmed quantitatively the significant tumor accumulation effect of the AuNR@IR780/ DOX-RGD-PEG NPs (Fig. 7a). ICP-MS results of $\mathrm{Au}$ in the tumor were further analyzed, and the tumor accumulation efficiency of the AuNR@IR780/DOX-RGD-PEG NPs was found to
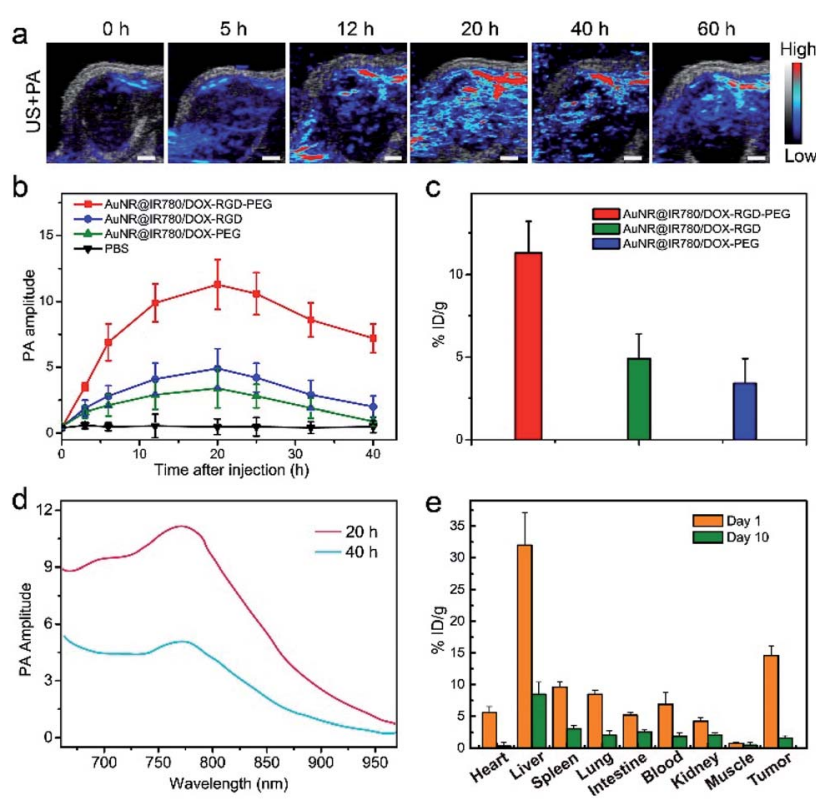

Fig. 7 In vivo PA imaging (a) PA amplitude (b) of the tumor treated with AuNRaIR780/DOX-RGD-PEG NPs at different post-injection time points. (Scale bar: $1 \mathrm{~mm}$ ). (c) Tumor accumulation efficiency of different samples at $24 \mathrm{~h}$ post-injection time points. (d) PA imaging curves of the samples in the tumor. (e) In vivo biodistribution of the hybrid NP in major organs and tissues of the tumor-bearing mice. 
be 11.5 ID per g, which was 2.4 and 3.5 times higher than that of the AuNR@IR780/DOX-RGD NPs (4.8 ID per g) and AuNR@IR780/DOX-PEG NPs (3.3 ID per g), respectively (Fig. 7c and $\mathrm{S} 12 \dagger)$. This was attributed to the fact that the existence of the PEG shell improved the in vivo circulation time of the NPs, and the pH-triggered shedding of PEG in the acidic tumor environment caused the RGD-functionalized NPs to accumulate in the targeted tumor region.

In order to examine the in vivo biodistribution of the AuNR@IR780/DOX-RGD-PEG NPs, in vivo PA imaging of the tumor at $40 \mathrm{~h}$ and $60 \mathrm{~h}$ post-injection was performed. The PA signal in the tumor was reduced, because the IR780-DOX shell was dissolved in the acidic environment of the tumor, the NP size was decreased, and the accumulated NPs could be efficiently removed from the tumor. The PA spectra of the tumor at $40 \mathrm{~h}$ post-injection was remarkably weaker than that at $20 \mathrm{~h}$ post-injection, further suggesting that some of accumulated theranostic NPs were removed from the tumor (Fig. 7d).

In the next step, the metabolism of the theranostic NPs was investigated by measuring the Au content in the main organs based on the ICP-MS results of Au. ${ }^{46}$ As it can be seen in Fig. 7e, most theranostic NPs were removed from the body. For example, the accumulated amount of NPs in the liver was reduced from $\sim 32 \%$ ID per $g$ to 8.2 ID per $g$. A high liver accumulation efficiency was also observed for the AuNR@IR780/DOX-RGD NPs or AuNR@IR780/DOX-PEG NPs treated tumor-bearing mice, however, which showed relative low tumor accumulation amount (Fig. S10†). The high accumulation effect and fast removal ability of the theranostic NPs guaranteed its great potential for in vivo therapy applications.

\section{In Vivo PA imaging guided synergistic chemo-photothermal therapy}

Encouraged by the high tumor accumulation, pH-responsive drug release, and excellent PTT effects of the AuNR@IR780/ DOX-RGD-PEG NP, its applicability for in vivo synergistic chemo-photothermal therapy was investigated. The theranostic AuNR@IR780/DOX-RGD-PEG NPs were intravenously injected into the mice through the tail vein when the tumor volume is $\sim 60 \mathrm{~mm}^{3}$. At $20 \mathrm{~h}$ post-injection, an $808 \mathrm{~nm}$ laser $\left(0.25 \mathrm{~W} \mathrm{~cm}^{-2}\right)$ was used to irradiate the whole tumor region and the tumor temperature was real-time recorded. It was found that the tumor temperature increased to $\sim 52{ }^{\circ} \mathrm{C}$ after a 5 min laser irradiation at a power density of $0.25 \mathrm{~W} \mathrm{~cm}^{-2}$ (Fig. 8a). Consistent with the lower tumor accumulation levels of the AuNR@IR780/DOX-RGD and AuNR@IR780/DOX-PEG NPs, the temperature of the tumors treated with these NPs increased to $\sim 44{ }^{\circ} \mathrm{C}$ and $\sim 42{ }^{\circ} \mathrm{C}$, respectively, which were lower than the tumor temperature with the AuNR@IR780/DOX-RGD-PEG NPs.

To further investigate the in vivo synergistic cancer therapy effect, U87MG tumor bearing mice were divided into the following six groups, with 5 mice in each experimental group: (1) PBS, (2) free DOX, (3) AuNR@IR780/DOX-RGD NPs, (4) AuNR@IR780/DOX-PEG NPs, (5) AuNR@IR780/DOX-RGD-PEG NPs, and (6) AuNR@IR780/DOX-RGD-PEG NPs with laser irradiation. As shown in Fig. 8b, the tumors in the mice of group 6
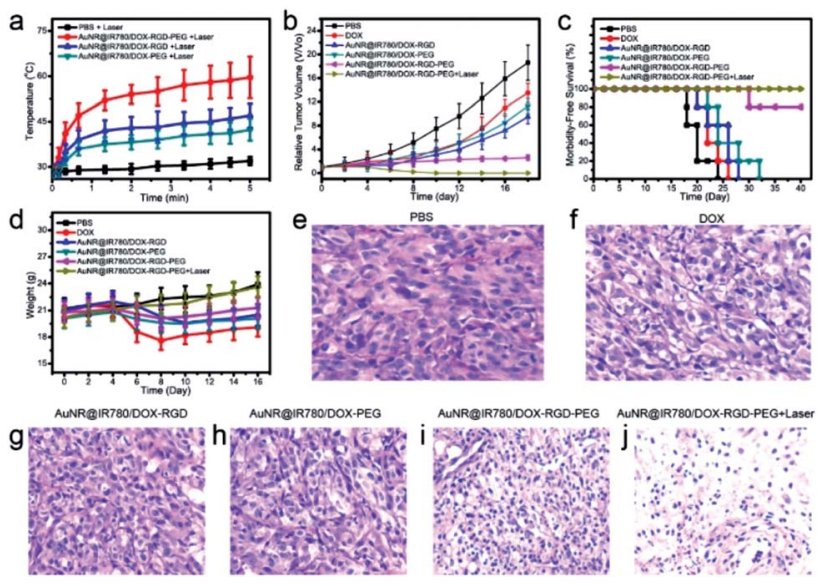

Fig. 8 (a) Temperature increase of the tumor after treatment with different samples and laser irradiation. (b) Relative tumor volume of the tumor-bearing mice in different treatment conditions. Average body weight (c) and the mice survive cure (d) of the mice in different groups. (e-j) Hematoxylin and eosin (H\&E) staining of the tumor tissue in different treatment groups.

were completely eliminated without regrowth, indicating an excellent synergistic chemo-photothermal effect of the theranostic NPs. For the tumors treated with theranostic NPs without laser irradiation (group 5), the tumor growth speed was instantly reduced but the tumors were not completely eliminated. Due to the relative low tumor accumulation levels of the AuNR@IR780/DOX-RGD and AuNR@IR780/DOX-PEG NPs, the tumors in groups 3 and 4 a relatively slow tumor growth compared to the control groups. The satisfactory therapeutic effect of the theranostic NPs was ascribed to the stimuliresponsive PEG shedding and the targeting effect of RGD, which lead to high tumor accumulation. However, the theranostic NP exhibited low therapy efficacy for the MCF-7 tumorbearing mice and free RGD pre-treated U87MG tumor-bearing mice (Fig. S13†).

In Fig. 8c, the survival rates of the mice in the 6 different treatment groups are demonstrated. The mice administered with AuNR@IR780/DOX-RGD-PEG NPs and laser irradiation survived over 40 days and no mice died. Furthermore, the average body weight of the mice in this group was slightly increased (Fig. 8d). However, nearly all the mice in the free DOX, AuNR@IR780/DOX-RGD NPs, and AuNR@IR780/DOX-PEG NPs groups died within one month, and their body weight was either decreased or increased very slowly (Fig. 8d). Moreover, hematoxylin and eosin (H\&E) images of tumor sections indicated that the tumor cells of the theranostic NPs and laser irradiation group were completely damaged, while in the control group the tumor cells were healthy and in the other groups (groups 3-5) the tumors were partially damaged, as displayed in Fig. $8 \mathrm{e}-\mathrm{j} \cdot{ }^{47,48}$ No obvious damage was find in the H\&E images of the main organs in the treatment group, suggesting the theranostic NP is biocompatible (Fig. S14 $\dagger$ ). These results confirmed that the theranostic NPs showed potential to be a strong candidate for the therapy of human glioblastoma. The excellent therapy effect of the suggested NP can be attributed to the high tumor 
accumulation efficiency due to PEG protection during in vivo circulation, the increased uptake of $\alpha_{v} \beta_{3}$-positive U87MG cancer cells after PEG shedding in the acidic tumor environment, and the localized pH-responsive DOX release in the lysosome and endosome. ${ }^{43}$

\section{Conclusion}

In summary, a PA imaging-activated and Trojan-horse like theranostic NP was developed, showing enhanced PA imaging performance and specific synergistic chemo-photothermal cancer therapy effect. Based on the experimental data, the generated electromagnetic field around the AuNR surface increased the light absorption efficiency of the IR780 coating shell. The results showed that the AuNR plays an antenna-like role causing a greater fraction of light to be absorbed by IR780. Moreover, an active targeting strategy was developed in order to cover the NP surface, which was conjugated with the RGD ligand, by co-conjugating a pH-cleavable PEG shield. Due to the PEG protection, the theranostic NPs demonstrated a long circulation time in the bloodstream and reduced non-specific uptake by healthy cells. In the tumor site, the PEG shedding, triggered by the characteristic tumor acidic environment, made the exposed RGD able to specifically bind to tumor cells in vivo. The theranostic NPs showed enhanced in vivo PA imaging performance, complete tumor elimination, and long survival time due to the active targeting strategy.

\section{Conflicts of interest}

There are no conflicts to declare.

\section{Acknowledgements}

This research was supported by National Natural Science Foundation of China (NSFC) projects (Grant number: 81871384, 81771869, 81571708, 81501506 and 21874024), the Research Fund of Science and Technology Department of Jilin Province (Grant number: 20160101001JC), the Norman Bethune Program of Jilin University (Grant number: 2015219) and the Hygiene Specific Subjects of Jilin Province (Grant number: 2018SCZ039).

\section{Notes and references}

1 Y. Lyu, J. Zeng, Y. Jiang, X. Zhen, T. Wang, S. Qiu, X. Lou, M. Gao and K. Pu, ACS Nano, 2018, 12, 1801-1810.

2 H. Moon, D. Kumar, H. Kim, C. Sim, J.-H. Chang, J.-M. Kim, H. Kim and D.-K. Lim, ACS Nano, 2015, 9, 2711-2719.

3 Y. Liu, N. Kang, J. Lv, Z. Zhou, Q. Zhao, L. Ma, Z. Chen, L. Ren and L. Nie, Adv. Mater., 2016, 28, 6411-6419.

4 J. Song, X. Yang, O. Jacobson, L. Lin, P. Huang, G. Niu, Q. Ma and X. Chen, ACS Nano, 2015, 9, 9199-9209.

5 Q. Miao, Y. Lyu, D. Ding and K. Pu, Adv. Mater., 2016, 28, 3662-3668.

6 W. Li, X. Sun, Y. Wang, G. Niu, X. Chen, Z. Qian and L. Nie, Biomed. Opt. Express, 2014, 5, 2679-2685.
7 X. Huang, J. Song, B. C. Yung, X. Huang, Y. Xiong and X. Chen, Chem. Soc. Rev., 2018, 47, 2873-2920.

8 L. S. Lin, J. Song, H. H. Yang and X. Chen, Adv. Mater., 2018, 30, 1704639.

9 J. Li, C. Xie, J. Huang, Y. Jiang, Q. Miao and K. Pu, Angew. Chem., Int. Ed. Engl., 2018, 57, 3995-3998.

10 Y. Lyu, J. Zeng, Y. Jiang, X. Zhen, T. Wang, S. Qiu, X. Lou, M. Gao and K. Pu, ACS Nano, 2018, 12, 1801-1810.

11 Q. Miao, Y. Lyu, D. Ding and K. Pu, Adv. Mater., 2016, 28, 3662-3668.

12 A. Kumar, S. Kim and J. M. Nam, J. Am. Chem. Soc., 2016, 138, 14509-14525.

13 Q. Miao and K. Pu, Bioconjugate Chem., 2016, 27, 2808-2823. 14 C. Xie, P. K. Upputuri, X. Zhen, M. Pramanik and K. Pu, Biomaterials, 2017, 119, 1-8.

15 J. V. Jokerst, A. J. Cole, D. Van de Sompel and S. S. Gambhir, ACS Nano, 2012, 6, 10366-10377.

16 A. Agarwal, S. W. Huang, M. O'Donnell, K. C. Day, M. Day, N. Kotov and S. Ashkenazi, J. Appl. Phys., 2007, 102, 064701. 17 H. Li, P. Zhang, L. P. Smaga, R. A. Hoffman and J. Chan, J. Am. Chem. Soc., 2015, 137, 15628-15631.

18 X. Tang, L. Tan, K. Shi, J. Peng, Y. Xiao, W. Li, L. Chen, Q. Yang and Z. Qian, Acta Pharm. Sin. B, 2018, 8, 587-601.

19 E. Liu, M. Zhang, H. Cui, J. Gong, Y. Huang, J. Wang, Y. Cui, W. Dong, L. Sun, H. He and V. C. Yang, Acta Pharm. Sin. B, 2018, 8, 956-968.

20 Q. Chen, X. Liu, J. Chen, J. Zeng, Z. Cheng and Z. Liu, Adv. Mater., 2015, 27, 6820-6827.

21 H. Hong, K. Yang, Y. Zhang, J. W. Engle, L. Feng, Y. Yang, T. R. Nayak, S. Goel, J. Bean, C. P. Theuer, T. E. Barnhart, Z. Liu and W. Cai, ACS Nano, 2012, 6, 2361-2370.

22 H. Moon, D. Kumar, H. Kim, C. Sim, J.-H. Chang, J.-M. Kim, H. Kim and D.-K. Lim, ACS Nano, 2015, 9, 2711-2719.

23 M. Li, L. Zhao, T. Zhang, Y. Shu, Z. He, Y. Ma, D. Liu and Y. Wang, Acta Pharm. Sin. B, 2018, 9, 421-432.

24 S. Pal, S. Harmsen, A. Oseledchyk, H.-T. Hsu and M. F. Kircher, Adv. Funct. Mater., 2017, 1606632.

25 Y. Peng, Z. Zhao, T. Liu, X. Li, X. Hu, X. Wei, X. Zhang and W. Tan, Angew. Chem., Int. Ed. Engl., 2017, 56, 10845-10849.

26 Z. Li, P. Huang, X. Zhang, J. Lin, S. Yang, B. Liu, F. Gao, P. Xi, Q. Ren and D. Cui, Mol. Pharm., 2010, 7, 94-104.

27 C. Wang, C. Bao, S. Liang, H. Fu, K. Wang, M. Deng, Q. Liao and D. Cui, Nanoscale Res. Lett., 2014, 9, 264.

28 W. Xu, T. Luo, P. Li, C. Zhou, D. Cui, B. Pang, Q. Ren and S. Fu, Int. J. Nanomed., 2012, 7, 915-924.

29 D. Ling, W. Park, S. J. Park, Y. Lu, K. S. Kim, M. J. Hackett, B. H. Kim, H. Yim, Y. S. Jeon, K. Na and T. Hyeon, J. Am. Chem. Soc., 2014, 136, 5647-5655.

30 Y. Yuan, Z. Ding, J. Qian, J. Zhang, J. Xu, X. Dong, T. Han, S. Ge, Y. Luo, Y. Wang, K. Zhong and G. Liang, Nano Lett., 2016, 16, 2686-2691.

31 J. Song, X. Yang, O. Jacobson, P. Huang, X. Sun, L. Lin, X. Yan, G. Niu, Q. Ma and X. Chen, Adv. Mater., 2015, 27, 4910-4917.

32 Z. Shen, J. Song, B. C. Yung, Z. Zhou, A. Wu and X. Chen, Adv. Mater., 2018, 30, e1704007. 
33 H. Kim, S. Beack, S. Han, M. Shin, T. Lee, Y. Park, K. S. Kim, A. K. Yetisen, S. H. Yun, W. Kwon and S. K. Hahn, Adv. Mater., 2018, 1701460.

34 C. Chu, H. Lin, H. Liu, X. Wang, J. Wang, P. Zhang, H. Gao, C. Huang, Y. Zeng, Y. Tan, G. Liu and X. Chen, Adv. Mater., 2017, 1605928.

35 J. G. Hinman, J. R. Eller, W. Lin, J. Li, J. Li and C. J. Murphy, J. Am. Chem. Soc., 2017, 139, 9851-9854.

36 M. F. Tsai, S. H. Chang, F. Y. Cheng, V. Shanmugam, Y. S. Cheng, C. H. Su and C. S. Yeh, ACS Nano, 2013, 7, 5330-5342.

37 J. Zhou, Y. Jiang, S. Hou, P. K. Upputuri, D. Wu, J. Li, P. Wang, X. Zhen, M. Pramanik, K. Pu and H. Duan, ACS Nano, 2018, 12, 2643-2651.

38 J. Song, X. Yang, Z. Yang, L. Lin, Y. Liu, Z. Zhou, Z. Shen, G. Yu, Y. Dai, O. Jacobson, J. Munasinghe, B. Yung, G. J. Teng and X. Chen, ACS Nano, 2017, 11, 6102-6113.

39 J. Song, F. Wang, X. Yang, B. Ning, M. G. Harp, S. H. Culp, S. Hu, P. Huang, L. Nie, J. Chen and X. Chen, J. Am. Chem. Soc., 2016, 138, 7005-7015.
40 J. Song, J. Zhou and H. Duan, J. Am. Chem. Soc., 2012, 134, 13458-13469.

41 L.-S. Lin, X. Yang, G. Niu, J. Song, H.-H. Yang and X. Chen, Nanoscale, 2016, 8, 2116-2122.

42 J. Song, Z. Fang, C. Wang, J. Zhou, B. Duan, L. Pu and H. Duan, Nanoscale, 2013, 5, 5816-5824.

43 Z. Shen, T. Chen, X. Ma, W. Ren, Z. Zhou, G. Zhu, A. Zhang, Y. Liu, J. Song, Z. Li, H. Ruan, W. Fan, L. Lin, J. Munasinghe, X. Chen and A. Wu, ACS Nano, 2017, 11, 10992-11004.

44 H. J. Li, J. Z. Du, J. Liu, X. J. Du, S. Shen, Y. H. Zhu, X. Wang, X. Ye, S. Nie and J. Wang, ACS Nano, 2016, 10, 6753-6761.

45 H.-J. Li, J.-Z. Du, X.-J. Du, C.-F. Xu, C.-Y. Sun, H.-X. Wang, Z.-T. Cao, X.-Z. Yang, Y.-H. Zhu, S. Nie and J. Wang, Proc. Natl. Acad. Sci. U. S. A., 2016, 113, 4164-4169.

46 J. Song, L. Pu, J. Zhou, B. Duan and H. Duan, ACS Nano, 2013, 7, 9947-9960.

47 Z. Shen, H. Wu, S. Yang, X. Ma, Z. Li, M. Tan and A. Wu, Biomaterials, 2015, 70, 1-11.

48 S. Shen, H. J. Li, K. G. Chen, Y. C. Wang, X. Z. Yang, Z. X. Lian, J. Z. Du and J. Wang, Nano Lett., 2017, 17, 38223829. 